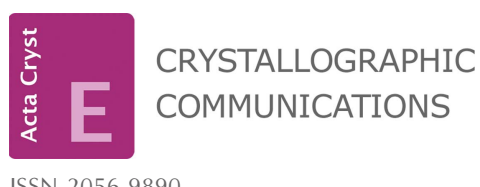

ISSN 2056-9890

Reçu le 1 avril 2016

Accepté le 10 mai 2016

Edité par P. Roussel, ENSCL, France

Keywords: crystal structure; alluaudite structure; framework; BVS; CHARDI.

CCDC reference: 1479074

Supporting information: this article has supporting information at journals.iucr.org/e

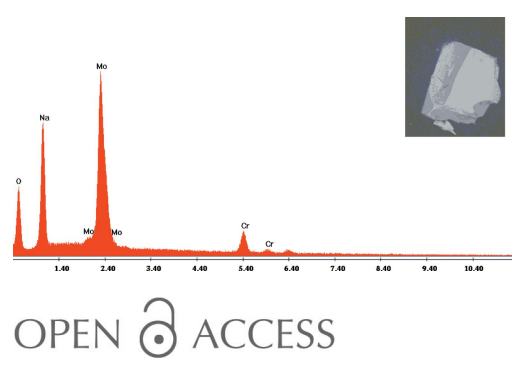

\section{Elaboration, étude structurale et analyse CHARDI et BVS d'une nouvelle variété $\beta-\mathrm{Na}{ }_{9} \mathrm{Cr}\left(\mathrm{MoO}_{4}\right)_{6}$ de type alluaudite}

\author{
Manel Sonni, Riadh Marzouki, Mohamed Faouzi Zid et Amira Souilem*
}

Laboratoire de Matériaux et Cristallochimie, Faculté des Sciences de Tunis, Université de Tunis El Manar, 2092 Manar II Tunis, Tunisia. *Correspondence e-mail: souilem_amira@yahoo.fr

The title compound, nonasodium chromium(III) hexakis[molybdate(VI)], $\beta$-Na $\mathrm{Na}^{-}$ $\mathrm{CrMo}_{6} \mathrm{O}_{24}$, was prepared by solid-state reactions. This alluaudite-type structure is constituted of infinite layers formed by links between $M_{2} \mathrm{O}_{10}(M=\mathrm{C} / \mathrm{Na})$ dimers and $\mathrm{MoO}_{4}$ tetrahedra. The $\mathrm{Na}^{+}$and $\mathrm{Cr}^{3+}$ cations are located in the same site with, respectively, 0.25 and 0.75 occupancies. The layers are connected to each other through $\mathrm{MoO}_{4}$ sharing corners, resulting an in open threedimensional framework with hexagonal-form cavities occupied by $\mathrm{Na}^{+}$cations. The proposed structural model is supported by charge-distribution (CHARDI) and bond-valence-sum (BVS) analysis. All atoms are on general positions except for one Mo, two Na (site symmetry 2) and another Na site (site symmetry $\overline{1})$. A comparison is made with the similar structures $\mathrm{Na}_{4} \mathrm{Co}\left(\mathrm{MoO}_{4}\right)_{3}$, $\mathrm{Na}_{2} \mathrm{Ni}\left(\mathrm{MoO}_{4}\right)_{2}, \mathrm{Cu}_{1.35} \mathrm{Fe}_{3}\left(\mathrm{PO}_{4}\right)_{3}$ and $\mathrm{NaAgFeMn}{ }_{2}\left(\mathrm{PO}_{4}\right)_{3}$.

\section{Contexte chimique}

La recherche de nouveaux matériaux pouvant être potentiellement des conducteurs ioniques ou bien des échangeurs d'ions, a conduit à s'intéresser aux composés à charpentes mixtes formées d'octaèdres $M_{6}(M=$ métal de transition biou trivalent) et de tétraèdres $\mathrm{MoO}_{4}$.

En effet, la jonction entre ces différents polyèdres conduit à des matériaux à charpentes ouvertes mixtes présentant de nombreuses propriétés physico-chimiques intéressantes qui sont en relation directe avec leurs structures cristallines, notamment: $\mathrm{LiFe}\left(\mathrm{MoO}_{4}\right)_{2}$ (van der Lee et al., 2008), $\mathrm{Na}_{2} \mathrm{Ni}\left(\mathrm{MoO}_{4}\right)_{2}$ (Klevtsova et al., 1991), $\mathrm{CsFe}\left(\mathrm{MoO}_{4}\right)_{2}(\mathrm{Baza}-$ rov et al., 2010), $\beta-\mathrm{NaFe}_{2}\left(\mathrm{MoO}_{4}\right)_{3}$ (Muessig et al., 2003), $\mathrm{K}_{2} \mathrm{Co}_{2}\left(\mathrm{MoO}_{4}\right)_{3}$ (Engel et al., 2009), $\mathrm{K}_{0.13} \mathrm{Na}_{3.87} \mathrm{MgMo}_{3} \mathrm{O}_{12}$ (Ennajeh et al., 2015). Ce domaine est loin d'être entièrement exploré et fait l'objet des travaux présentant des intérêts fondamentaux qu'appliqués (Savina et al., 2014; Namsaraeva et al., 2011). C'est dans ce cadre que nous avons exploré les systèmes $A-\mathrm{Cr}-\mathrm{Mo}-\mathrm{O}$ ( $A=$ cation monovalent) dans lesquels nous avons précédemment caractérisé une seule phase intéressante: $\mathrm{Na}_{9} \mathrm{Cr}\left(\mathrm{MoO}_{4}\right)_{6}$ de symétrie hexagonale [la forme $\alpha-\mathrm{Na}_{9} \mathrm{Cr}\left(\mathrm{MoO}_{4}\right)_{6}$; Dridi et al., 2015]. Un nouveau composé de symétrie monoclinique a été synthétisé par réaction à l'état solide. Le mode de préparation, la détermination de la structure par diffraction des rayons-X sur monocristal et la validation des données, réalisée par les deux modèles CHARDI et BVS, seront présentés dans ce travail. La nouvelle variété $\beta$ - $\mathrm{Na}_{9} \mathrm{Cr}\left(\mathrm{MoO}_{4}\right)_{6}$, bien qu'elle appartient à la famille des Alluaudites, présente une formule différente de celle généralement adoptée pour cette série de composés: 
$A A^{\prime} M M^{\prime} X_{3} \mathrm{O}_{12}(A, M=$ métaux; $A=$ mono, $M=$ bi- ou trivalent et $X=\mathrm{Mo}, \mathrm{P}, \mathrm{As})$.

\section{Description de la structure}

L'unité structurale dans le composé $\beta$ - $\mathrm{Na}_{9} \mathrm{CrMo}_{6} \mathrm{O}_{24}$ est constituée de deux tétraèdres $\mathrm{MoO}_{4}$ et d'un octaèdre $\mathrm{MO}_{6}(M$ $=\mathrm{Cr} 1 / \mathrm{Na} 1)$ partageant des sommets oxygène. La compensation de charge est assurée par les cations $\mathrm{Na}^{+}$(Fig. 1). La jonction des différents polyèdres, par ponts mixtes $\mathrm{Mo}-\mathrm{O}-$ $M 1(M 1=\mathrm{Cr} 1 / \mathrm{Na} 1)$ et par arêtes entre octaèdres, engendre des couches qui se lient à leur tour par insertion de tétraèdres $\mathrm{Mo} 2 \mathrm{O}_{4}$ et conduit à une charpente tridimensionnelle. Chaque couche peut être décrite comme un assemblage de deux chaînes classiques de type $M 1 \mathrm{Mo}^{\mathrm{O}} \mathrm{O}_{8}(M 1=\mathrm{Cr} 1 / \mathrm{Na} 1)$. En effet, deux chaînes adjacentes se regroupent par mise en commun d'arêtes entre octaèdres pour conduire à des chaînes doubles de type $M 1_{2} \mathrm{Mo1}_{2} \mathrm{O}_{14}$. Ces dernières se connectent moyennant des ponts mixtes de types $\mathrm{Mo1}-\mathrm{O}-M$ pour donner des couches disposées parallèlement au plan $b c$ (Fig. 2). Au sein d'une couche, les dimères appartenant à deux chaînes doubles adjacentes sont orientés perpendiculairement les uns par rapport aux autres. Cette disposition a favorisé l'orientation des tétraèdres $\mathrm{Mo}_{10}$, en trans, et a conduit à la jonction des couches par insertion des tétraèdres $\mathrm{Mo}_{2} \mathrm{O}_{4}$ entre elles par l'établissement des ponts mixtes $\mathrm{Mo} 2-\mathrm{O}-M_{2} \mathrm{O}_{10}$

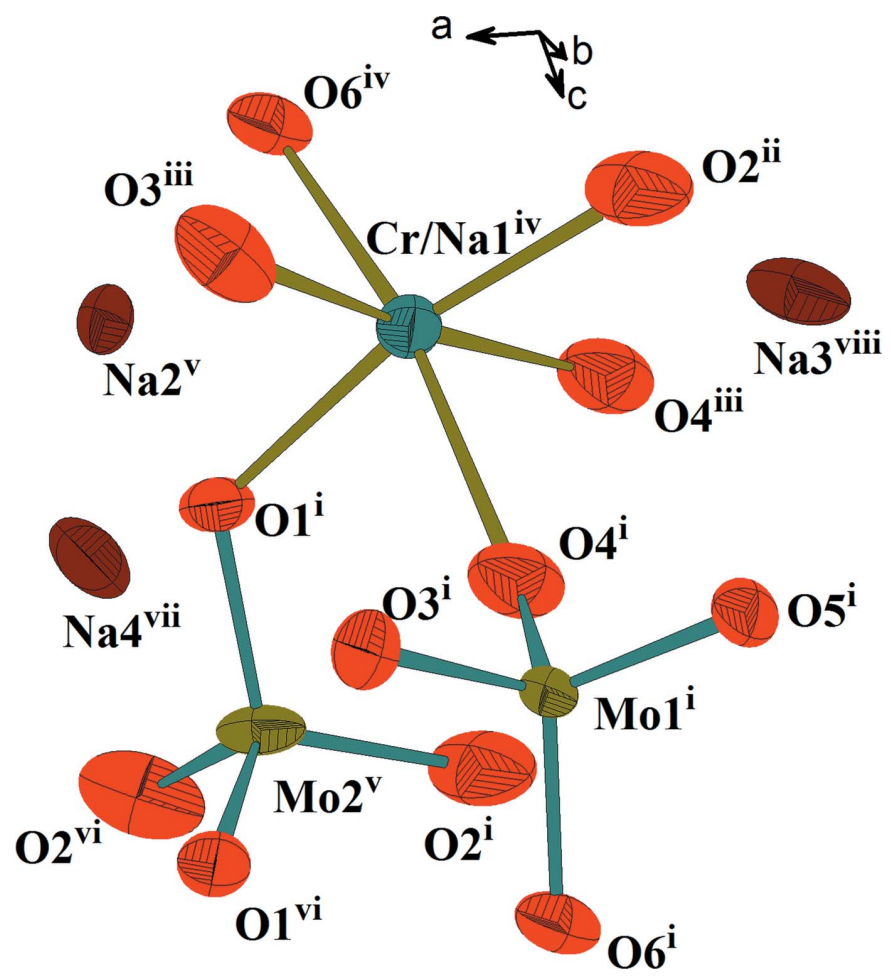

Figure 1

Unité structurale mettant en évidence les polyèdres de coordination dans l'unité asymétrique de $\beta-\mathrm{Na}_{9} \mathrm{CrMo}_{6} \mathrm{O}_{24}$. Les éllipsoïdes ont été définis avec $50 \%$ de probabilité. [Codes de symétrie: (i) $-\frac{1}{2}+x, \frac{1}{2}-y, \frac{1}{2}+z$; (ii) $1-x, y, \frac{1}{2}-z$; (iii) $-\frac{1}{2}+x,-\frac{1}{2}+y, z$; (iv) $-\frac{1}{2}+x,-\frac{1}{2}-y,-\frac{1}{2}+z$; (v) $\frac{1}{2}-x$, $\frac{1}{2}-y, 1-z ;\left(\right.$ vi) $\frac{3}{2}-x, \frac{1}{2}-y, 1-z ;$ (vii) $\frac{1}{2}+x,-\frac{1}{2}+y, z ;$ (viii) $\frac{1}{2}-x,-\frac{1}{2}+y$, $\frac{1}{2}-z$.]

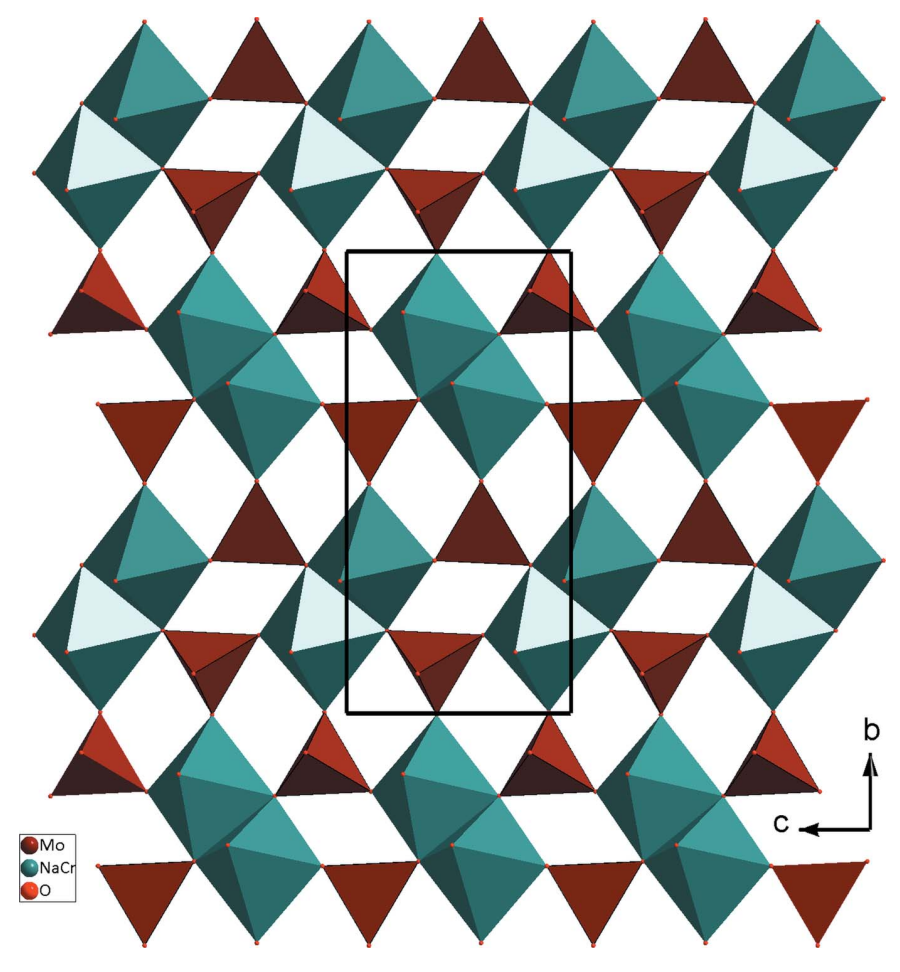

Figure 2

Projection d'une couche parallèlement au plan $b c$.

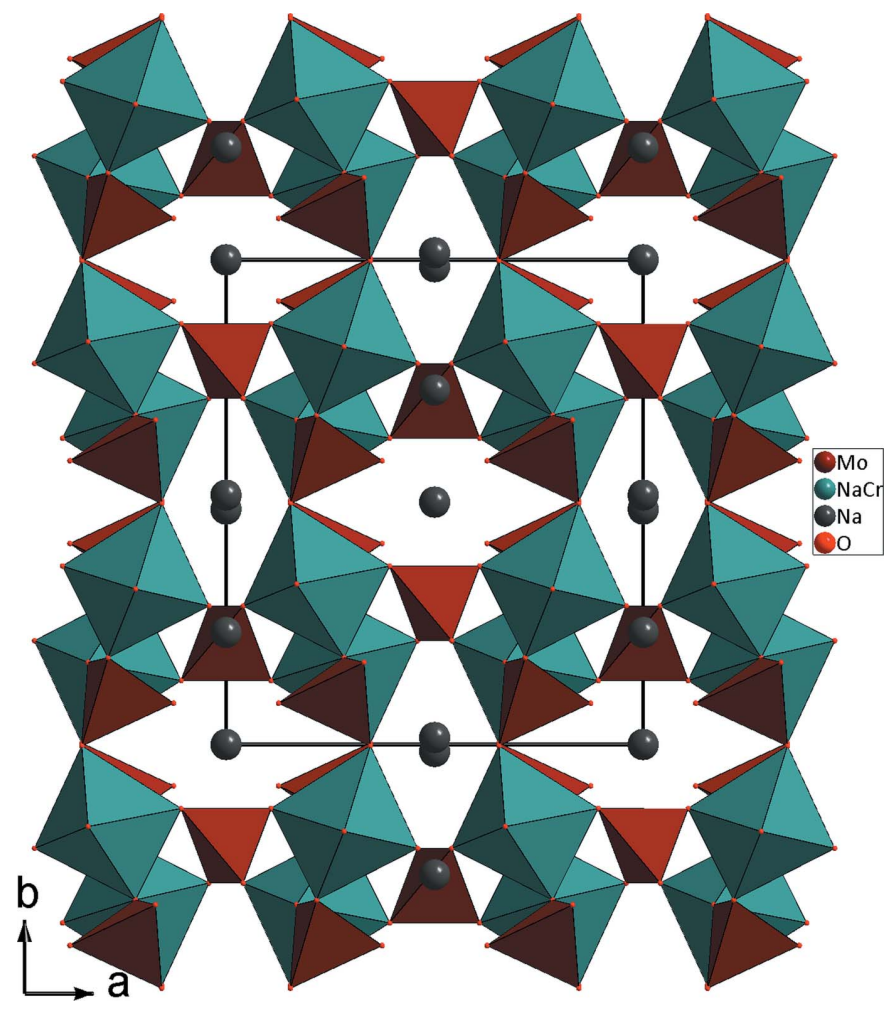

Figure 3

Projection de la structure de $\beta-\mathrm{Na}_{9} \mathrm{CrMo}_{6} \mathrm{O}_{24}$, selon $c$, mettant en évidence les cavités où résident les cation $\mathrm{Na}^{+}$. 


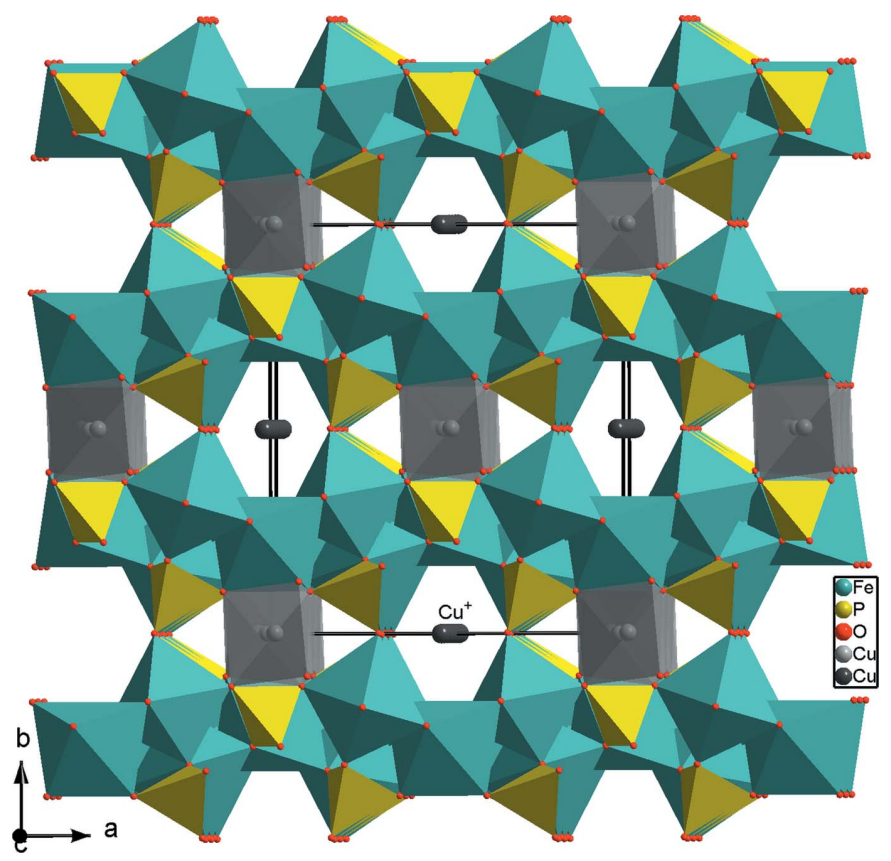

Figure 4

Projection de la structure de $\mathrm{Cu}_{1,35} \mathrm{Fe}_{3}\left(\mathrm{PO}_{4}\right)_{3}$, selon $c$, montrant l'emplacement des ions $\mathrm{Cu}^{2+}$ et $\mathrm{Cu}^{+}$dans le réseau.

$(M=\mathrm{Cr} 1 / \mathrm{Na} 1)$. Il en résulte une charpente tridimensionnelle laissant libre des cavités, à section hexagonale, où résident les cations alcalins $\mathrm{Na}^{+}$(Fig. 3).

\section{Enquête de base de données}

Cette variété, que nous avons appelé $\beta-\mathrm{Na}_{9} \mathrm{CrMo}_{6} \mathrm{O}_{24}$, est isoformulaire à celle précédemment obtenue $\alpha-\mathrm{Na}_{9} \mathrm{CrMo}_{6} \mathrm{O}_{24}$ (Dridi et al., 2015). Ces deux structures révèlent une différence nette. En effet, la forme bêta cristallise dans le système monoclinique, groupe d'espace $C 2 / c$ et appartient à la famille des alluaudites possédant une charpentes tridimensionnelle alors que la forme alpha se caractérise par une structure à charpente unidimensionnelle et cristallise dans le système rhomboédrique, groupe d'espace $R \overline{3} c$ avec les paramètres de maille suivants: $a=14,707$ et $c=19,175 \AA$. Un examen bibliographique des matériaux rencontrés dans la littérature et ayant une formulation générale de type $A_{x} M X_{3} \mathrm{O}_{12}(M=\mathrm{Co}$, $\mathrm{Ni}, \mathrm{Mn}$, Fe et $X=\mathrm{Mo}, \mathrm{P})$, révèle une certaine similitude dans les structures de $\mathrm{Na}_{4} \mathrm{Co}\left(\mathrm{MoO}_{4}\right)_{3}$ (Nasri et al., 2014), $\mathrm{Na}_{2} \mathrm{Ni}\left(\mathrm{MoO}_{4}\right)_{2}$ (Klevtsova et al., 1991), $\mathrm{Cu}_{1,35} \mathrm{Fe}_{3}\left(\mathrm{PO}_{4}\right)_{3}$ (Warner et al., 1993) et $\mathrm{NaAgFeMn}{ }_{2}\left(\mathrm{PO}_{4}\right)_{3}$ (Daidouh et al., 2002). Ces matériaux diffèrent essentiellement par l'occupation et la répartition des sites octaédriques. En effet, dans la structure de $\mathrm{Cu}_{1,35} \mathrm{Fe}_{3}\left(\mathrm{PO}_{4}\right)_{3}$ (Fig. 4) l'ion $\mathrm{Cu}^{2+}$ occupe le canal en remplaçant l'ion sodium $\mathrm{Na}^{+}$situé à l'origine de la maille et le cation $\mathrm{Cu}^{+}$joue le rôle de l'ion alcalin $\mathrm{Na}^{+}$dans le composé étudié $\beta-\mathrm{Na}_{9} \mathrm{Cr}\left(\mathrm{MoO}_{4}\right)_{6}$. Alors que dans la structure de $\mathrm{NaAgFeMn}\left(\mathrm{PO}_{4}\right)_{3}$, les ions $\mathrm{Mn}^{2+}$ forment des octaèdres qui s'associent par ponts mixtes de type $\mathrm{Mn}-\mathrm{O}-\mathrm{P}$ ou bien
Tableau 1

Longueurs de liaison sélectionnés $(\AA)$.

\begin{tabular}{llll}
\hline $\mathrm{Mo1}-\mathrm{O} 5$ & $1.736(4)$ & $\mathrm{Cr} 1-\mathrm{O} 3^{\mathrm{v}}$ & $2.240(5)$ \\
$\mathrm{Mo1}-\mathrm{O} 6$ & $1.742(4)$ & $\mathrm{Cr} 1-\mathrm{O} 4^{\mathrm{iii}}$ & $2.335(5)$ \\
$\mathrm{Mo1}-\mathrm{O} 3$ & $1.762(4)$ & $\mathrm{Na} 2-\mathrm{O} 1^{\text {vi }}$ & $2.375(3)$ \\
$\mathrm{Mo1}-\mathrm{O} 4$ & $1.762(4)$ & $\mathrm{Na} 2-\mathrm{O} 6^{\text {vii }}$ & $2.401(4)$ \\
$\mathrm{Mo} 2-\mathrm{O} 1^{\mathrm{i}}$ & $1.750(3)$ & $\mathrm{Na} 2-\mathrm{O} 5^{\text {vii }}$ & $2.472(4)$ \\
$\mathrm{Mo} 2-\mathrm{O} 1^{\text {ii }}$ & $1.750(3)$ & $\mathrm{Na} 3-\mathrm{O} 5$ & $2.494(3)$ \\
$\mathrm{Mo} 2-\mathrm{O} 2^{\text {ii }}$ & $1.756(5)$ & $\mathrm{Na} 3-\mathrm{O} 2^{\text {ix }}$ & $2.532(4)$ \\
$\mathrm{Cr} 1-\mathrm{O} 2^{\text {iii }}$ & $2.132(6)$ & $\mathrm{Na} 3-\mathrm{O} 5^{\mathrm{i}}$ & $2.683(4)$ \\
$\mathrm{Cr} 1-\mathrm{O} 1^{\text {iv }}$ & $2.153(4)$ & $\mathrm{Na} 4-\mathrm{O} 3^{\text {ii }}$ & $2.513(5)$ \\
$\mathrm{Cr} 1-\mathrm{O} 6$ & $2.181(4)$ & $\mathrm{Na} 4-\mathrm{O} 3^{\mathrm{x}}$ & $2.575(5)$ \\
$\mathrm{Cr} 1-\mathrm{O} 4^{\text {iv }}$ & $2.210(4)$ & & \\
\hline
\end{tabular}

Symmetry codes: (i) $-x+1, y,-z+\frac{1}{2}$; (ii) $x-1, y, z$; (iii) $-x+\frac{3}{2},-y+\frac{1}{2},-z+1$; (iv) $x, y, z+1$; (v) $x,-y+1, z+\frac{1}{2}$; (vi) $x-1, y, z+1$; (vii) $-x+1, y,-z+\frac{3}{2}$; (viii) $-x+\frac{1}{2},-y+\frac{1}{2},-z+1$; (ix) $x-\frac{1}{2},-y+\frac{1}{2}, z-\frac{1}{2}$; (x) $x-1,-y+1, z+\frac{1}{2}$.

$\mathrm{Mn}-\mathrm{O}-M(M=\mathrm{Fe} / \mathrm{Mn})$ et renforcent la jonction des couches dans la charpente tridimensionnelle (Fig. 5).

Si on se limite, à une sphère de coordination de rayon égal à $3 \AA$ moyennant le programme DIAMOND (Brandenburg \& Putz, 2001), on montre que les polyèdres $\mathrm{Na}_{2} \mathrm{O}_{6}, \mathrm{Na}_{3} \mathrm{O}_{6}$ et $\mathrm{Na}_{4} \mathrm{O}_{4}$ sont irréguliers (tableau 1), mais les moyennes des distances $\mathrm{Na}-\mathrm{O}$, dans la structure sont conformes à celles rencontrées dans la littérature. De plus, le calcul des différentes valences des liaisons utilisant la formule empirique de Brown (Brown \& Altermatt, 1985) vérifie bien les valeurs de charges des ions: Mo1 (6,09), Mo2 (6,15), Cr1/Na1 (1,69), Na2 $(1,12), \mathrm{Na} 3(0,87), \mathrm{Na} 4(0,76)$, attendues dans la phase étudiée.

Le modèle structural proposé, particulièrement la distribution au site $M 1(\mathrm{Cr} 1 / \mathrm{Na} 1)$, est confirmé par les deux modèles de validation: la somme des valences de liaisons BVS (Brown, 2002; Adams, 2003) et la méthode de distribution de charges CHARDI (Nespolo et al., 2001; Nespolo, 2001) (tableau 2).

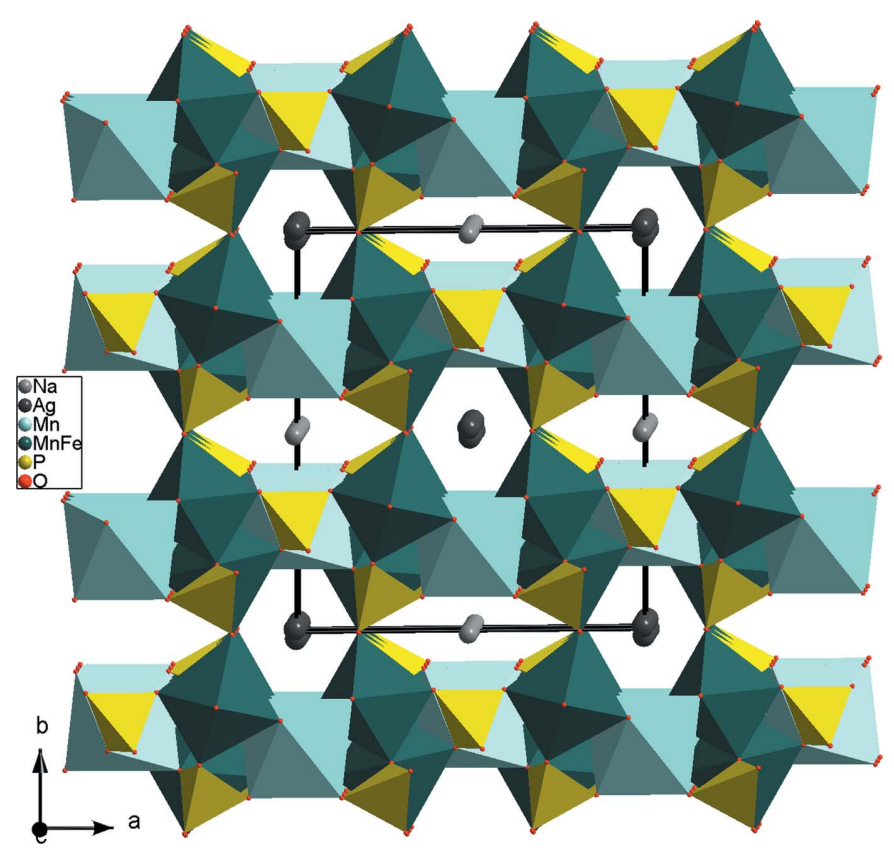

Figure 5

Projection de la structure de $\mathrm{NaAgFeMn}\left(\mathrm{PO}_{4}\right)_{3}$, selon $c$, mettant en évidence la jonction des couches dans la charpente tridimensionnelle. 
Tableau 2

Analyse CHARDI et BVS des cations dans le composé $\beta-\mathrm{Na}_{9} \mathrm{CrMo}_{6} \mathrm{O}_{24}$.

\begin{tabular}{llllllll}
\hline Cation & $q(i) \cdot \operatorname{sof}(i)$ & $Q(i)$ & $V(i)$ & $\mathrm{CN}(i)$ & $\mathrm{ECoN}(i)$ & $d_{\text {moy }}$ & $d_{\text {med }}$ \\
\hline Mo1 & 6,000 & 6,130 & 6,0988 & 4 & 3,99 & 1,7502 & 1,7507 \\
Mo2 & 6,000 & 6,150 & 6,1532 & 4 & 4,00 & 1,7525 & 1,7524 \\
$M(\mathrm{Cr} / \mathrm{Na})$ & 1,50 & 1,300 & 1,6875 & 6 & 5,82 & 2,2085 & 2,2084 \\
Na2 & 1,000 & 1,010 & 1,1166 & 6 & 5,94 & 2,4158 & 2,4159 \\
Na3 & 1,000 & 1,010 & 0,8719 & 6 & 6,14 & 2,5699 & 2,5695 \\
Na4 & 1,000 & 0,970 & 0,7578 & 4 & 5,02 & 2,5441 & 2,5443 \\
\hline
\end{tabular}

$q(i)=$ nombre d'oxydation formel; $\operatorname{sof}(i)=$ taux d'occupation du site; $\mathrm{CN}(i)=$ nombre de coordination classique; $Q(i)=$ charge calculée; $V(i)=$ valence calculée; $\mathrm{ECoN}(i)=$ nombre de coordination effectif; $d_{\text {moy }}(i)=$ distance arithmétique moyenne; $d_{\text {med }}(i)=$ distance pondérée moyenne; $\sigma_{\text {cat }}=$ facteur de dispersion sur les charges cationiques; $\sigma_{\text {cat }}=$ $\left[\Sigma_{\mathrm{i}}\left(q_{\mathrm{i}}-Q_{\mathrm{i}}\right)^{2} / N-1\right]^{1 / 2}=0,025$.

Les valeurs de charges calculées $Q(i)$ et de valences $V(i)$ sont en bon accord avec les degrés d'oxydation pondérés par les taux d'occupation. Le facteur de dispersion $\sigma_{\text {cat }}$ (Nespolo, 2001) qui mesure la déviation des charges cationiques calculées est égal à 2,5\%.

\section{Synthèse et cristallisation}

Au cours de l'investigation des diagrammes $A-\mathrm{Mo}-\mathrm{Cr}-\mathrm{O}(A=$ $\mathrm{Li}, \mathrm{Na}, \mathrm{Ag})$ un nouveau composé de fomulation $\beta-\mathrm{Na}_{9} \mathrm{CrMo}_{6} \mathrm{O}_{24}$ a été élaboré. Les cristaux ont été obtenus par voie sèche en broyant dans un mortier en agate les réactifs $\mathrm{NaNO}_{3}, \mathrm{Cr}\left(\mathrm{NO}_{3}\right)_{3} \cdot 9 \mathrm{H}_{2} \mathrm{O}$ et $\left(\mathrm{NH}_{4}\right)_{6} \mathrm{Mo}_{7} \mathrm{O}_{24} \cdot 4 \mathrm{H}_{2} \mathrm{O}$ dans les rapports molaires Na:Cr:Mo égalent à 6:1:4, respectivement. Le mélange obtenu a subit une calcination à $673 \mathrm{~K}$ afin

Tableau 3

Détails expérimentaux.

\begin{tabular}{|c|c|}
\hline \multicolumn{2}{|l|}{ Données crystallines } \\
\hline Formule chimique & $\mathrm{Na}_{9} \mathrm{CrMo}_{6} \mathrm{O}_{24}$ \\
\hline$M_{\mathrm{r}}$ & 1218,55 \\
\hline Système cristallin, groupe d'espace & Monoclinique, $C 2 / c$ \\
\hline Température (K) & 298 \\
\hline$a, b, c(\AA)$ & $12,655(2), 13,578(2), 7,1405(8)$ \\
\hline$\beta\left({ }^{\circ}\right)$ & $112,58(2)$ \\
\hline$V\left(\AA^{3}\right)$ & $1132,9(3)$ \\
\hline$Z$ & 2 \\
\hline Type de rayonnement & Мo $K \alpha$ \\
\hline$\mu\left(\mathrm{mm}^{-1}\right)$ & 3,96 \\
\hline Taille des cristaux (mm) & $0,28 \times 0,22 \times 0,14$ \\
\hline \multicolumn{2}{|l|}{ Collection de données } \\
\hline Diffractomètre & Enraf-Nonius CAD-4 \\
\hline Correction d'absorption & $\psi$ scan (North et al., 1968) \\
\hline$T_{\min }, T_{\max }$ & $0,356,0,574$ \\
\hline $\begin{array}{l}\text { Nombre de réflexions mesurées, } \\
\text { indépendantes et observées } \\
{[I>2 \sigma(I)]}\end{array}$ & $2518,1231,1065$ \\
\hline$R_{\text {int }}$ & 0,023 \\
\hline$(\sin \theta / \lambda)_{\max }\left(\AA^{-1}\right)$ & 0,638 \\
\hline \multicolumn{2}{|l|}{ Affinement } \\
\hline$R\left[F^{2}>2 \sigma\left(F^{2}\right)\right], w R\left(F^{2}\right), S$ & $0,028,0,080,1,06$ \\
\hline Nombre de réflexions & 1231 \\
\hline Nombre de paramètres & 97 \\
\hline$\Delta \rho_{\max }, \Delta \rho_{\min }\left(\mathrm{e} \AA^{-3}\right)$ & $0,92,-0,98$ \\
\hline
\end{tabular}

Programmes informatiques: CAD-4 EXPRESS (Duisenberg, 1992; Macíček \& Yordanov, 1992), XCAD4 (Harms \& Wocadlo, 1995), SHELXS97 et SHELXL97 (Sheldrick, 2008), DIAMOND (Brandenburg \& Putz, 2001), WinGX (Farrugia, 2012) et publCIF (Westrip, 2010).

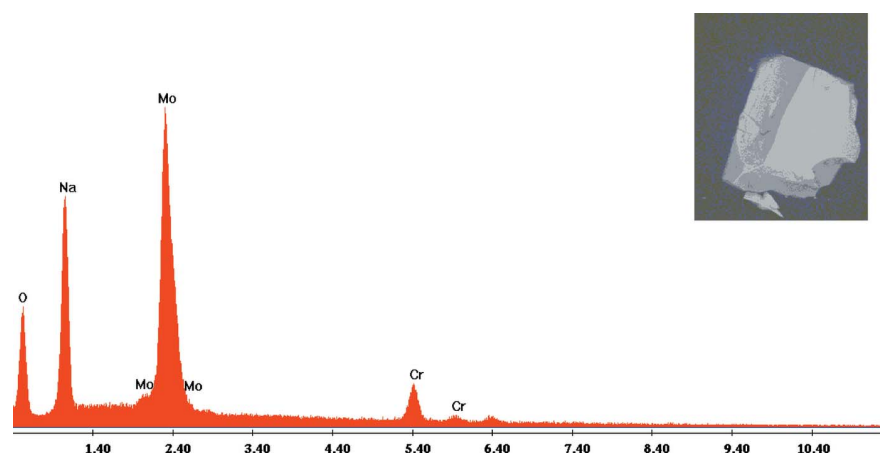

Figure 6

Analyse qualitative au MEB et morphologie d'un cristal de la variété $\beta-\mathrm{Na}_{9} \mathrm{CrMo}_{6} \mathrm{O}_{24}$.

d'éliminer les produits volatils notamment: $\mathrm{NO}_{2}, \mathrm{NH}_{3}$ et $\mathrm{H}_{2} \mathrm{O}$. Ensuite, la poudre résiduelle a subit un broyage fin puis remis dans le four à une température proche de la fusion à $973 \mathrm{~K}$ pendant trois jours pour favoriser la germination et la croissance des cristaux. Après refroidissement du four, des cristaux de forme parallélépipédique et de taille optimale pour la collecte des données, ont été obtenus. Une analyse qualitative au MEB de marque FEI et de type Quanta 200 confirme la présence des différents éléments chimiques attendus: $\mathrm{Mo}, \mathrm{Cr}$, $\mathrm{Na}$ et l'oxygène (Fig. 6).

\section{Affinement}

Un cristal sélectionné sous microscope polarisant, de bonne qualité, a servi pour les mesures des intensités (tableau 3). Un examen des facteurs géométriques montre que la distance $M 1-\mathrm{O}$ égale à 2,208 $\AA$ ( $M 1=\mathrm{Cr} 1 / \mathrm{Na} 1)$ s'avère une moyenne entre celles $\mathrm{Cr} 1-\mathrm{O}$ et $\mathrm{Na} 1-\mathrm{O}$ rencontrées dans la littérature. Un affinement des contraintes EADP et EXYZ ainsi que SUMP autorisées par le programme SHELXL97 (Sheldrick, 2008), a conduit vers des taux d'occupation du Cr1 et Na1 égaux à 0,244 (6) et 0,755 (7) respectivement. Les conditions de la neutralité électrique pour le matériau nous ont encouragé à fixer ces derniers à $25 \%$ pour le $\mathrm{Cr} 1$ et $75 \%$ pour le Na1. A la fin de la résolution, les densités d'électrons maximum et minimum restants dans la Fourier-différence sont acceptables et sont situées respectivement à $0,78 \AA$ de Mo1 et $0,89 \AA$ de Mo2. De plus, l'affinement final conduit à des ellipsoïdes bien définis.

\section{Acknowledgements}

Les auteurs remercient le Ministère de l'Enseignement Supérieur et de la Recherche Scientifiques de la Tunisie pour le financement du laboratoire LMC LR01ES11.

\section{Références}

Adams, S. (2003). softBV. University of Göttingen, Germany. http:// kristall.uni-mki. gwdg. de/softBV/

Bazarov, B. G., Namsaraeva, T. V., Klevtsova, R. F., Anshits, A. G., Vereshchagina, T. A., Glinskaya, L. A., Fedorov, K. N. \& Bazarova, Zh. G. (2010). Kristallografiya, 55, 634-636. 
Brandenburg, K. \& Putz, H. (2001). DIAMOND. Crystal Impact GbR, Bonn, Allemagne.

Brown, I. D. (2002). The Chemical Bond in Inorganic Chemistry - The Bond Valence Model. IUCr Monographs on Crystallography, 12. Oxford University Press.

Brown, I. D. \& Altermatt, D. (1985). Acta Cryst. B41, 244-247.

Daidouh, A., Durio, C., Pico, C., Veiga, M. L., Chouaibi, N. \& Ouassini, A. (2002). Solid State Sci. 4, 541-548.

Dridi, W., Ennajeh, I. \& Zid, M. F. (2015). Acta Cryst. E71, 435-439.

Duisenberg, A. J. M. (1992). J. Appl. Cryst. 25, 92-96.

Engel, J. M., Ahsbahs, H., Fuess, H. \& Ehrenberg, H. (2009). Acta Cryst. B65, 29-35.

Ennajeh, I., Georges, S., Ben Smida, Y., Guesmi, A., Zid, M. F. \& Boughazala, H. (2015). RSC Adv. 5, 38918-38925.

Farrugia, L. J. (2012). J. Appl. Cryst. 45, 849-854.

Harms, K. \& Wocadlo, S. (1995). XCAD4. Université de Marburg, Allemagne.

Klevtsova, R. F., Borisov, S. V., Bliznyuk, N. A., Glinskaya, L. A. \& Klevtsov, P. V. (1991). Struct. Chem. 32, 127-136.

Lee, A. van der, Beaurain, M. \& Armand, P. (2008). Acta Cryst. C64, i1-i4.
Macíček, J. \& Yordanov, A. (1992). J. Appl. Cryst. 25, 73-80.

Muessig, E., Bramnik, K. G. \& Ehrenberg, H. (2003). Acta Cryst. B59, 611-616.

Namsaraeva, T., Bazarov, B., Mikhailova, D., Kuratieva, N., Sarapulova, A., Senyshyn, A. \& Ehrenberg, H. (2011). Eur. J. Inorg. Chem. 2011, 2832-2841.

Nasri, R., Fakhar Bourguiba, N., Zid, M. F. \& Driss, A. (2014). Acta Cryst. E70, i47-i48.

Nespolo, M. (2001). CHARDI-IT, CRM². Université Henri Poincaré Nancy I, France.

Nespolo, M., Ferraris, G., Ivaldi, G. \& Hoppe, R. (2001). Acta Cryst. B57, 652-664.

North, A. C. T., Phillips, D. C. \& Mathews, F. S. (1968). Acta Cryst. A24, 351-359.

Savina, A. A., Solodovnikov, S. F., Belov, D. A., Basovich, O. M., Solodovnikova, Z. A., Pokholok, K. V., Stefanovich, S., Lazoryak, B. I. \& Khaikina, E. G. (2014). J. Solid State Chem. 220, 217-220. Sheldrick, G. M. (2008). Acta Cryst. A64, 112-122.

Warner, T. E., Milius, W. \& Maier, J. (1993). J. Solid State Chem. 106, 301-309.

Westrip, S. P. (2010). J. Appl. Cryst. 43, 920-925. 


\section{supporting information}

Acta Cryst. (2016). E72, 833-837 [https://doi.org/10.1107/S205698901600774X]

Elaboration, étude structurale et analyse CHARDI et BVS d'une nouvelle variété $\beta-\mathrm{Na}{ }_{9} \mathrm{Cr}\left(\mathrm{MoO}_{4}\right)_{6}$ de type alluaudite

\section{Manel Sonni, Riadh Marzouki, Mohamed Faouzi Zid et Amira Souilem}

Computing details

Data collection: CAD-4 EXPRESS (Duisenberg, 1992; Macíček \& Yordanov, 1992); cell refinement: CAD-4 EXPRESS

(Duisenberg, 1992; Macíček \& Yordanov, 1992); data reduction: XCAD4 (Harms \& Wocadlo, 1995); program(s) used to solve structure: SHELXS97 (Sheldrick, 2008); program(s) used to refine structure: SHELXL97 (Sheldrick, 2008);

molecular graphics: DIAMOND (Brandenburg \& Putz, 2001); software used to prepare material for publication: WinGX (Farrugia, 2012) and publCIF (Westrip, 2010).

Nonasodium chromium(III) hexakis[molybdate(VI)]

Crystal data

$\mathrm{Na}_{9} \mathrm{CrMo}_{6} \mathrm{O}_{24}$

$M_{r}=1218.55$

Monoclinic, $C 2 / c$

Hall symbol: $-\mathrm{C} 2 \mathrm{yc}$

$a=12.655$ (2) $\AA$

$b=13.578(2) \AA$

$c=7.1405(8) \AA$

$\beta=112.58(2)^{\circ}$

$V=1132.9(3) \AA^{3}$

$Z=2$

$F(000)=1134$

$D_{\mathrm{x}}=3.572 \mathrm{Mg} \mathrm{m}^{-3}$

Mo $K \alpha$ radiation, $\lambda=0.71073 \AA$

Cell parameters from 25 reflections

$\theta=10-15^{\circ}$

$\mu=3.96 \mathrm{~mm}^{-1}$

$T=298 \mathrm{~K}$

Prism, red

$0.28 \times 0.22 \times 0.14 \mathrm{~mm}$

\section{Data collection}

Enraf-Nonius CAD-4 diffractometer

Radiation source: fine-focus sealed tube

Graphite monochromator

$\omega / 2 \theta$ scans

Absorption correction: $\psi$ scan

(North et al., 1968)

$T_{\min }=0.356, T_{\max }=0.574$

2518 measured reflections

1231 independent reflections

1065 reflections with $I>2 \sigma(I)$

$R_{\text {int }}=0.023$

$\theta_{\text {max }}=27.0^{\circ}, \theta_{\text {min }}=2.3^{\circ}$

$h=-16 \rightarrow 12$

$k=-1 \rightarrow 17$

$l=-9 \rightarrow 9$

2 standard reflections every $120 \mathrm{~min}$ intensity decay: $1.3 \%$

Refinement

Refinement on $F^{2}$

Least-squares matrix: full

$R\left[F^{2}>2 \sigma\left(F^{2}\right)\right]=0.028$

$w R\left(F^{2}\right)=0.080$

$S=1.06$

1231 reflections

97 parameters
0 restraints

Primary atom site location: structure-invariant direct methods

Secondary atom site location: difference Fourier map

$w=1 /\left[\sigma^{2}\left(F_{\mathrm{o}}^{2}\right)+(0.0433 P)^{2}+5.0898 P\right]$

where $P=\left(F_{\mathrm{o}}^{2}+2 F_{\mathrm{c}}^{2}\right) / 3$ 
$(\Delta / \sigma)_{\max }<0.001$

$\Delta \rho_{\max }=0.92$ e $\AA^{-3}$

$\Delta \rho_{\min }=-0.98$ e $\AA^{-3}$
Extinction correction: SHELXL97 (Sheldrick, 2008), $\mathrm{Fc}^{*}=\mathrm{kFc}\left[1+0.001 \mathrm{xFc}^{2} \lambda^{3} / \sin (2 \theta)\right]^{-1 / 4}$

Extinction coefficient: $0.0012(2)$

\section{Special details}

Geometry. All esds (except the esd in the dihedral angle between two 1.s. planes) are estimated using the full covariance matrix. The cell esds are taken into account individually in the estimation of esds in distances, angles and torsion angles; correlations between esds in cell parameters are only used when they are defined by crystal symmetry. An approximate (isotropic) treatment of cell esds is used for estimating esds involving l.s. planes.

Refinement. Refinement of $\mathrm{F}^{2}$ against ALL reflections. The weighted R-factor $\mathrm{wR}$ and goodness of fit $\mathrm{S}$ are based on $\mathrm{F}^{2}$, conventional R-factors $R$ are based on $F$, with $F$ set to zero for negative $F^{2}$. The threshold expression of $F^{2}>2 \operatorname{sigma}\left(\mathrm{F}^{2}\right)$ is used only for calculating R-factors(gt) etc. and is not relevant to the choice of reflections for refinement. R-factors based on $\mathrm{F}^{2}$ are statistically about twice as large as those based on F, and R- factors based on ALL data will be even larger.

Fractional atomic coordinates and isotropic or equivalent isotropic displacement parameters $\left(\AA^{2}\right)$

\begin{tabular}{llllll}
\hline & $x$ & $y$ & $z$ & $U_{\text {iss } * / U_{\text {eq }}}$ & Occ. $(<1)$ \\
\hline Mo1 & $0.77009(3)$ & $0.39149(3)$ & $0.37667(6)$ & $0.02349(17)$ & \\
Mo2 & 0.0000 & $0.21336(4)$ & 0.2500 & $0.0280(2)$ & \\
Cr1 & $0.78758(12)$ & $0.34023(11)$ & $0.8756(2)$ & $0.0267(3)$ & 0.25 \\
Na1 & $0.78758(12)$ & $0.34023(11)$ & $0.8756(2)$ & $0.0267(3)$ & 0.75 \\
Na2 & 0.0000 & $0.2313(2)$ & 0.7500 & $0.0244(5)$ & \\
Na3 & 0.5000 & 0.5000 & 0.0000 & $0.0409(8)$ & \\
Na4 & 0.0000 & $0.5144(4)$ & 0.7500 & $0.0591(12)$ & \\
O1 & $0.9590(3)$ & $0.2858(3)$ & $0.0309(5)$ & $0.0284(7)$ & \\
O2 & $0.8920(5)$ & $0.1320(3)$ & $0.2490(7)$ & $0.0557(13)$ & \\
O3 & $0.8458(4)$ & $0.5028(3)$ & $0.4008(7)$ & $0.0494(12)$ & \\
O4 & $0.7828(4)$ & $0.3206(4)$ & $0.1796(6)$ & $0.0471(11)$ & \\
O5 & $0.6258(3)$ & $0.4147(3)$ & $0.3165(6)$ & $0.0302(8)$ & \\
O6 & $0.8308(3)$ & $0.3307(3)$ & $0.6084(5)$ & $0.0340(8)$ & \\
& & & &
\end{tabular}

Atomic displacement parameters $\left(\AA^{2}\right)$

\begin{tabular}{lllllll}
\hline & $U^{11}$ & $U^{22}$ & $U^{33}$ & $U^{12}$ & $U^{13}$ & $U^{23}$ \\
\hline $\mathrm{Mo} 1$ & $0.0186(2)$ & $0.0331(3)$ & $0.0158(2)$ & $-0.00152(16)$ & $0.00336(16)$ & $0.00283(14)$ \\
$\mathrm{Mo} 2$ & $0.0463(4)$ & $0.0182(3)$ & $0.0140(3)$ & 0.000 & $0.0054(2)$ & 0.000 \\
$\mathrm{Cr} 1$ & $0.0274(8)$ & $0.0318(8)$ & $0.0209(7)$ & $-0.0042(6)$ & $0.0092(6)$ & $-0.0020(5)$ \\
$\mathrm{Na} 1$ & $0.0274(8)$ & $0.0318(8)$ & $0.0209(7)$ & $-0.0042(6)$ & $0.0092(6)$ & $-0.0020(5)$ \\
$\mathrm{Na} 2$ & $0.0216(13)$ & $0.0264(13)$ & $0.0291(14)$ & 0.000 & $0.0142(11)$ & 0.000 \\
$\mathrm{Na} 3$ & $0.052(2)$ & $0.0244(14)$ & $0.0282(15)$ & $0.0021(13)$ & $-0.0041(14)$ & $-0.0012(12)$ \\
$\mathrm{Na} 4$ & $0.0232(17)$ & $0.120(4)$ & $0.0291(17)$ & 0.000 & $0.0045(13)$ & 0.000 \\
$\mathrm{O} 1$ & $0.0319(18)$ & $0.0332(19)$ & $0.0195(16)$ & $0.0033(15)$ & $0.0092(14)$ & $0.0057(14)$ \\
$\mathrm{O} 2$ & $0.073(3)$ & $0.035(2)$ & $0.042(3)$ & $-0.014(2)$ & $0.002(2)$ & $0.0113(19)$ \\
$\mathrm{O} 3$ & $0.038(2)$ & $0.056(3)$ & $0.047(3)$ & $-0.018(2)$ & $0.009(2)$ & $0.015(2)$ \\
O4 & $0.042(2)$ & $0.071(3)$ & $0.028(2)$ & $0.012(2)$ & $0.0122(18)$ & $-0.001(2)$ \\
O5 & $0.0226(18)$ & $0.0288(18)$ & $0.035(2)$ & $0.0034(14)$ & $0.0067(15)$ & $0.0084(15)$ \\
O6 & $0.032(2)$ & $0.039(2)$ & $0.0249(19)$ & $0.0100(16)$ & $0.0037(15)$ & $0.0038(15)$ \\
\end{tabular}


Geometric parameters $\left(\AA,{ }^{o}\right)$

\begin{tabular}{|c|c|c|c|}
\hline Mo1-O5 & $1.736(4)$ & $\mathrm{Na} 2-\mathrm{O} 1^{\mathrm{i}}$ & $2.375(4)$ \\
\hline Mo1-O6 & $1.742(4)$ & $\mathrm{Na} 2-\mathrm{O}^{\mathrm{vii}}$ & $2.401(4)$ \\
\hline Mo1-O3 & $1.762(4)$ & $\mathrm{Na} 2-\mathrm{O}^{\mathrm{ii}}$ & $2.401(4)$ \\
\hline Mo1-O4 & $1.762(4)$ & $\mathrm{Na} 2-\mathrm{O} 5^{\mathrm{viii}}$ & $2.472(4)$ \\
\hline $\mathrm{Mo} 2-\mathrm{O} 1^{\mathrm{i}}$ & $1.750(3)$ & $\mathrm{Na} 2-\mathrm{O} 5^{\mathrm{ix}}$ & $2.472(4)$ \\
\hline $\mathrm{Mo} 2-\mathrm{O}^{\mathrm{ii}}$ & $1.750(3)$ & $\mathrm{Na} 3-\mathrm{O} 5$ & $2.494(3)$ \\
\hline $\mathrm{Mo} 2-\mathrm{O} 2^{\mathrm{ii}}$ & $1.756(5)$ & $\mathrm{Na} 3-\mathrm{O}^{\mathrm{x}}$ & $2.494(3)$ \\
\hline $\mathrm{Mo} 2-\mathrm{O} 2^{\mathrm{i}}$ & $1.756(5)$ & $\mathrm{Na} 3-\mathrm{O} 2^{\mathrm{xi}}$ & $2.532(4)$ \\
\hline $\mathrm{Cr} 1-\mathrm{O} 2^{\mathrm{iii}}$ & $2.132(6)$ & $\mathrm{Na} 3-\mathrm{O} 2^{\mathrm{xii}}$ & $2.532(4)$ \\
\hline $\mathrm{Cr} 1-\mathrm{O} 1^{\mathrm{iv}}$ & $2.153(4)$ & $\mathrm{Na} 3-\mathrm{O} 5^{\mathrm{i}}$ & $2.683(4)$ \\
\hline $\mathrm{Cr} 1-\mathrm{O} 6$ & $2.181(4)$ & $\mathrm{Na} 3-\mathrm{O} 5^{\mathrm{xiii}}$ & $2.683(4)$ \\
\hline $\mathrm{Cr} 1-\mathrm{O} 4^{\mathrm{iv}}$ & $2.210(4)$ & $\mathrm{Na} 4-\mathrm{O} 3^{\mathrm{ii}}$ & $2.513(5)$ \\
\hline $\mathrm{Cr} 1-\mathrm{O}^{\mathrm{v}}$ & $2.240(5)$ & $\mathrm{Na} 4-\mathrm{O} 3^{\mathrm{vii}}$ & $2.513(5)$ \\
\hline $\mathrm{Cr} 1-\mathrm{O} 4^{\mathrm{iii}}$ & $2.335(5)$ & $\mathrm{Na} 4-\mathrm{O} 3^{\text {xiv }}$ & $2.575(5)$ \\
\hline $\mathrm{Na} 2-\mathrm{O} 1^{\mathrm{vi}}$ & $2.375(3)$ & $\mathrm{Na} 4-\mathrm{O}^{\mathrm{xv}}$ & $2.575(5)$ \\
\hline $\mathrm{O} 5-\mathrm{Mo1}-\mathrm{O} 6$ & $111.06(18)$ & $\mathrm{O} 1^{\mathrm{iv}}-\mathrm{Cr} 1-\mathrm{O} 6$ & $83.61(14)$ \\
\hline $\mathrm{O} 5-\mathrm{Mo} 1-\mathrm{O} 3$ & $110.5(2)$ & $\mathrm{O} 2^{\mathrm{iii}}-\mathrm{Cr} 1-\mathrm{O} 4^{\mathrm{iv}}$ & 90.27 (19) \\
\hline $\mathrm{O} 6-\mathrm{Mo} 1-\mathrm{O} 3$ & $106.8(2)$ & $\mathrm{O} 1^{\mathrm{iv}}-\mathrm{Cr} 1-\mathrm{O} 4^{\mathrm{iv}}$ & $81.65(16)$ \\
\hline $\mathrm{O} 5-\mathrm{Mo1}-\mathrm{O} 4$ & $108.13(19)$ & $\mathrm{O} 6-\mathrm{Cr} 1-\mathrm{O} 4^{\mathrm{iv}}$ & $164.22(18)$ \\
\hline $\mathrm{O} 6-\mathrm{Mo1}-\mathrm{O} 4$ & $111.0(2)$ & $\mathrm{O} 2^{\mathrm{iii}-\mathrm{Cr} 1-\mathrm{O}^{\mathrm{v}}}$ & 97.49 (17) \\
\hline $\mathrm{O} 3-\mathrm{Mo} 1-\mathrm{O} 4$ & $109.4(2)$ & $\mathrm{O} 1^{\mathrm{iv}}-\mathrm{Cr} 1-\mathrm{O}^{\mathrm{v}}$ & 92.85 (16) \\
\hline $\mathrm{O} 1^{\mathrm{i}}-\mathrm{Mo} 2-\mathrm{O} 1^{\mathrm{ii}}$ & $111.6(2)$ & $\mathrm{O} 6-\mathrm{Cr} 1-\mathrm{O}^{\mathrm{v}}$ & 86.64 (17) \\
\hline $\mathrm{O} 1^{\mathrm{i}}-\mathrm{Mo} 2-\mathrm{O} 2^{\mathrm{ii}}$ & $108.7(2)$ & $\mathrm{O} 4^{\mathrm{iv}}-\mathrm{Cr} 1-\mathrm{O}^{\mathrm{v}}$ & 99.67 (19) \\
\hline $\mathrm{O} 1^{\mathrm{ii}}-\mathrm{Mo} 2-\mathrm{O} 2^{\mathrm{ii}}$ & $112.77(19)$ & $\mathrm{O} 2^{\mathrm{iii}}-\mathrm{Cr} 1-\mathrm{O} 4^{\mathrm{iii}}$ & 79.54 (17) \\
\hline $\mathrm{O} 1^{\mathrm{i}}-\mathrm{Mo} 2-\mathrm{O} 2^{\mathrm{i}}$ & 112.77 (19) & $\mathrm{O} 1^{\mathrm{iv}}-\mathrm{Cr} 1-\mathrm{O} 4^{\mathrm{iii}}$ & $90.56(15)$ \\
\hline $\mathrm{O} 1^{\mathrm{ii}-}-\mathrm{Mo} 2-\mathrm{O} 2^{\mathrm{i}}$ & $108.7(2)$ & $\mathrm{O} 6-\mathrm{Cr} 1-\mathrm{O} 4^{\mathrm{iii}}$ & $90.24(15)$ \\
\hline 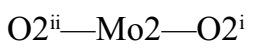 & $102.0(4)$ & $\mathrm{O} 4^{\mathrm{iv}}-\mathrm{Cr} 1-\mathrm{O} 4^{\mathrm{iii}}$ & $84.30(17)$ \\
\hline $\mathrm{O} 2^{\mathrm{iii}}-\mathrm{Cr} 1-\mathrm{O}^{\mathrm{iv}}$ & $167.83(16)$ & $\mathrm{O}^{\mathrm{v}}-\mathrm{Cr} 1-\mathrm{O} 4^{\mathrm{iii}}$ & $175.10(17)$ \\
\hline $\mathrm{O} 2^{\mathrm{iii}-\mathrm{Cr} 1-\mathrm{O} 6}$ & $103.32(18)$ & & \\
\hline
\end{tabular}

Symmetry codes: (i) $-x+1, y,-z+1 / 2$; (ii) $x-1, y, z$; (iii) $-x+3 / 2,-y+1 / 2,-z+1$; (iv) $x, y, z+1$; (v) $x,-y+1, z+1 / 2$; (vi) $x-1, y, z+1$; (vii) $-x+1, y,-z+3 / 2$; (viii) $-x+1 / 2,-y+1 / 2,-z+1$; (ix) $x-1 / 2,-y+1 / 2, z+1 / 2$; (x) $-x+1,-y+1,-z$; (xi) $x-1 / 2,-y+1 / 2, z-1 / 2$; (xii) $-x+3 / 2, y+1 / 2,-z+1 / 2$; (xiii) $x,-y+1, z-1 / 2$; (xiv) $x-1,-y+1, z+1 / 2 ;(\mathrm{xv})-x+1,-y+1,-z+1$. 